\title{
Topological properties of some nanostructures
}

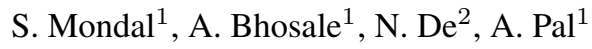 \\ ${ }^{1}$ Department of mathematics, NIT Durgapur, India, \\ ${ }^{2}$ Department of Basic Sciences and Humanities (Mathematics), \\ Calcutta Institute of Engineering and Management, Kolkata, India \\ souravmath94@gmail.com, bhosaleakshay78@gmail.com,de.nilanjan@rediffmail.com, anita.buie@gmail.com
}

DOI 10.17586/2220-8054-2020-11-1-14-24

\begin{abstract}
Topological indices are numerical values associated with chemical constitution describing the structures of chemical compounds and helping to predict different physicochemical properties. In this report, some newly designed topological descriptors, namely, neighborhood Zagreb index $\left(M_{N}\right)$, neighborhood version of Forgotten topological index $\left(F_{N}\right)$, modified neighborhood version of Forgotten topological index $\left(F_{N}^{*}\right)$, neighborhood version of second Zagreb index $\left(M_{2}^{*}\right)$, neighborhood version of hyper Zagreb index $\left(H M_{N}\right)$ are obtained for the $T U R C_{4} C_{8}(S)$, armchair nanotube $T U A C_{6}$, V-phenylenic nanotube $V P H X[m, n]$, and V-phenylenic nanotori $V P H Y[m, n]$.
\end{abstract}

Keywords: Topological indices, $T U R C_{4} C_{8}(S)$, armchair nanotube $\left(T U A C_{6}\right)$, V-phenylenic nanotube $(V P H X[m, n])$, V-phenylenic nanotori ( $V P H Y[m, n])$.

Received: 30 December 2019

Revised: 3 January 2020

\section{Introduction}

We consider only molecular graphs throughout this article. By molecular graph [1-3], we mean a simple connected graph in which nodes are supposed to be atoms and edges are chemical bonds. The vertex and edge sets of a graph $G$ are represented here by $V(G)$ and $E(G)$, respectively. The degree of a vertex $v$ on a graph $G$, denoted by $d_{G}(v)$, is the total number of edges associated with $v$. Moreover, we define

$$
\delta_{G}(v)=\sum_{u \in N_{G}(v)} \operatorname{deg}_{G}(u)
$$

where

$$
N_{G}(v)=\{u \in V(G): u v \in E(G)\} .
$$

The chemical graph theory has a significant impact on the chemical science development. Chemical graph theory is a part of mathematical chemistry that uses graph theory for mathematically modeling chemical phenomena. In this field, a leading tool is topological index. A real valued mapping considering graphs as arguments is called a graph invariant if it gives same value to isomorphic graphs. In chemical graph theory, the graph invariants are named as topological indices. Topological indices play key role in QSPR/QSAR study. Topological indices interpret chemical compound structures and help to predict certain physicochemical properties such as entropy, boiling point, acentric factor, vaporization enthalpy, etc. Among different types of topological indices, degree based topological indices have prominent role in this research area. For some well-known degree based topological indices, readers are referred to [4-8]. In [9,10], some new neighborhood degree based indices are presented having good correlations with entropy and acentric factor. They are defined as follows.

The neighborhood Zagreb index is denoted by $M_{N}(G)$ and is defined as:

$$
M_{N}(G)=\sum_{v \in V(G)} \delta_{G}(v)^{2}
$$

Neighborhood version of Forgotten topological index is denoted by $F_{N}(G)$ and is defined as:

$$
F_{N}(G)=\sum_{v \in V(G)} \delta_{G}(v)^{3} .
$$

Modified neighborhood version of Forgotten topological index is denoted by $F_{N}^{*}(G)$ and is defined as:

$$
F_{N}^{*}(G)=\sum_{u v \in E(G)}\left[\delta_{G}(u)^{2}+\delta_{G}(v)^{2}\right] .
$$


Neighborhood version of second Zagreb index is denoted by $M_{2}^{*}(G)$ and is defined by:

$$
M_{2}^{*}(G)=\sum_{u v \in E(G)}\left[\delta_{G}(u) \delta_{G}(v)\right] .
$$

Neighborhood version of hyper Zagreb index is denoted by $H M_{N}(G)$ and is defined by:

$$
H M_{N}(G)=\sum_{u v \in E(G)}\left[\delta_{G}(u)+\delta_{G}(v)\right]^{2} .
$$

A nanostructure is an intermediate object between microscopic and molecular structures. It is a molecular-scale product obtained from engineering. The most important class of such materials is the carbon nanotubes. Carbon nanotubes (CNTs) are carbon allotropes with cylindrical molecular structures, having diameters ranging from a few nanometers and lengths to several millimeters. Nanotubes are categorized as single-walled (SWNTs) and multiwalled (MWNTs) nanotubes. Researchers have found topological descriptors for various nanotube and nanotori. In [11], topological properties of $T U R C_{4} C_{8}(S)$ are investigated. Jiang et al. determined topological index of Vphenylenic nanotubes and V-phenylenic nanotori in [12]. Topological properties of armchair polyhex nanotube are discussed in [13]. For more discussion on this topic, readers are referred to [14-21]. Inspired by these works, we have derived $M_{N}, F_{N}, F_{N}^{*}, M_{2}^{*}$, and $H M_{N}$ indices for $T U R C_{4} C_{8}(S)$, armchair polyhex nanotube $T U A C_{6}$, Vphenylenic nanotube $V P H X[m, n]$, and V-phenylenic nanotori $V P H Y[m, n]$. Moreover, we have compared these indices graphically.

\section{Motivation}

The correlation coefficient $(r)$ of topological indices with different physicochemical properties for a benchmark data set is determined to check the utility of the indices in QSPR/QSAR analysis. According to the International Academy of Mathematical chemistry, an index is considered to be useful if $r^{2} \geq 0.8$. In [9, 10], the chemical applicability of the indices $M_{N}, F_{N}, F_{N}^{*}, M_{2}^{*}$, and $H M_{N}$ are studied taking octane isomers as data set. The $r^{2}$ values of those indices with entropy are $0.907,0.88,0.868,0.899$ and 0.88 , respectively. The $r^{2}$ values of those indices with acentric factor are $0.989,0.989,0.952,0.971$ and 0.961 , respectively. The aforesaid indices are therefore effective in QSPR/QSAR analysis with powerful accuracy. In addition, their isomer discrimination ability also remarkable [9, 10] in comparison with the other degree based indices. With the help of nanotechnology, many new materials and devices are in progress with a wide range of applications in medicine, electronics and computers. Motivated by the importance of topological indices and the nanotechnology, we intend to compute the aforementioned indices for some nanostructures which are described in the next section.

\section{Preliminaries}

The 2D and 3D lattice of $T U R C_{4} C_{8}(S)$ nanotube are shown in Fig. 1. We consider $m n$ numbers of $C_{8}$ and $C_{4}$ cycles in the $2 \mathrm{D}$ lattice of $T U R C_{4} C_{8}(S)$ nanotube. We denote this graph by $T U C_{4} C_{8}[m, n]$. From Fig. 1, it is clear that this graph has $8 m n+2 m$ nodes and $12 m n+m$ edges.
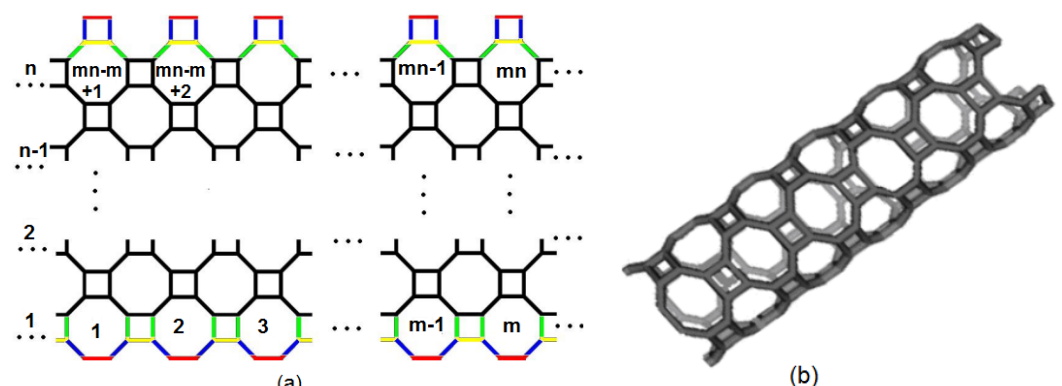

(a)

(b)

FIG. 1. (a) The $2 \mathrm{D}$ and (b)the $3 \mathrm{D}$ lattice of $T U C_{4} C_{8}[m, n]$ nanotube

We consider a class of armchair polyhex nano tubes $T U A C_{6}[m, n]$ having $m$ and $n$ numbers of hexagons in each rows and columns, respectively. The molecular graph of $T U A C_{6}[m, n]$ is depicted in the Fig. 2. We can say from Fig. 2, that $m$ is even for all $n \in N$. This nanotube has $2 m n+2 m$ and $3 m n+2 m$ numbers of nodes and edges respectively. 

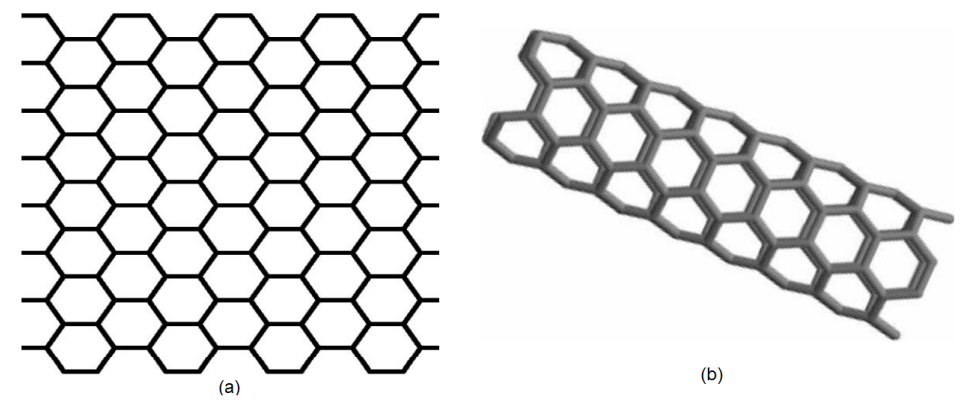

(b)

FIG. 2. (a) The $2 \mathrm{D}$ and (b)the $3 \mathrm{D}$ lattice of Armchair polyhex nanotubes $T U A C_{6}$

Also we consider V-phenylenic nanotube and V-phenylenic nanotori whose 2D lattices are depicted in Figs. 3 and 4. Phenylenes are polycyclic conjugated molecules, made of $C_{4}$ and $C_{6}$ such that every $C_{4}$ is adjacent to two $C_{6}$ and lies between two $C_{6}$. No two $C_{6}$ are mutually adjacent. Each $C_{6}$ is adjacent to only two $C_{4}$ cycles.

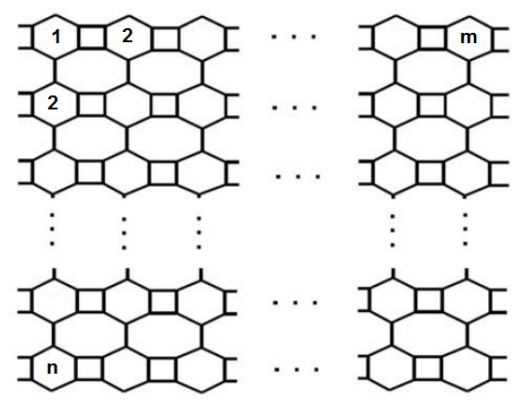

FIG. 3. The molecular graph of V-phenylenic nanotube $V P H X[m, n]$

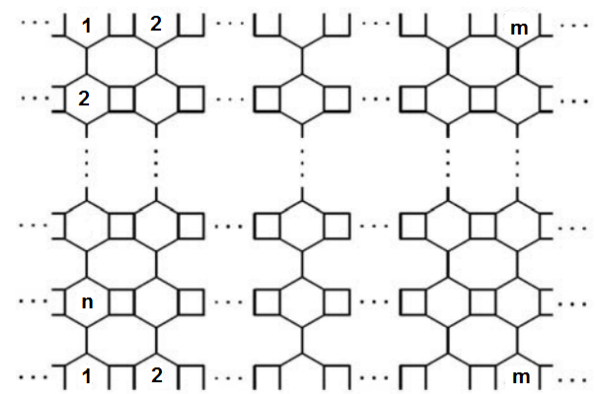

FIG. 4. The molecular graph of V-phenylenic nanotori $V P H Y[m, n]$

\section{Main results}

In this section, our goal is to compute aforesaid indices for $T U C_{4} C_{8}[m, n]$, armchair polyhex nanotube $T U A C_{6}$, V-phenylenic nanotube $V P H X[m, n]$, and V-phenylenic nanotori $V P H Y[m, n]$. Following Figs. 1, 2, 3, and 4, first we obtain vertex and edge partitions of nanotubes and nanotori discussed above and then proceed for main theorems.

The vertex and edge partitions for $T U C_{4} C_{8}[m, n]$ nanotube are given in Table 1 and 2, respectively. The vertex and edge partitions for $T U A C_{6}[m, n]$ nanotube are given in Table 3 and 4 , respectively. The vertex and edge partitions for $V P H X[m, n]$ nanotube are given in Table 5 and 6 , respectively. 
TABLE 1. Vertex partition of $T U C_{4} C_{8}[m, n]$

\begin{tabular}{|c|c|c|c|}
\hline$\delta_{G}(u)$ & 5 & 8 & 9 \\
\hline Frequency & $4 m$ & $4 m$ & $8 m n-6 m$ \\
\hline
\end{tabular}

TABLE 2. Edge partition of $T U C_{4} C_{8}[m, n]$

\begin{tabular}{|c|c|c|c|c|c|}
\hline$\left(\delta_{G}(u), \delta_{G}(v)\right)$ & $(5,5)$ & $(5,8)$ & $(8,8)$ & $(8,9)$ & $(9,9)$ \\
\hline Frequency & $2 m$ & $4 m$ & $2 m$ & $4 m$ & $12 m n-11 m$ \\
\hline
\end{tabular}

TABLE 3. Vertex partition of $T U A C_{6}[m, n]$

\begin{tabular}{|c|c|c|c|}
\hline$\delta_{G}(u)$ & 5 & 8 & 9 \\
\hline Frequency & $2 m$ & $2 m$ & $2 m(n-1)$ \\
\hline
\end{tabular}

TABLE 4. Edge partition of $T U A C_{6}[m, n]$

\begin{tabular}{|c|c|c|c|c|c|}
\hline$\left(\delta_{G}(u), \delta_{G}(v)\right)$ & $(5,5)$ & $(5,8)$ & $(8,8)$ & $(8,9)$ & $(9,9)$ \\
\hline Frequency & $m$ & $2 m$ & $m$ & $2 m$ & $m(3 n-4 m)$ \\
\hline
\end{tabular}

TABLE 5. Vertex partition of $V P H X[m, n]$

\begin{tabular}{|c|c|c|c|}
\hline$\delta_{G}(u)$ & 6 & 8 & 9 \\
\hline Frequency & $2 m$ & $4 m$ & $6 m n-6 m$ \\
\hline
\end{tabular}

TABLE 6. Edge partition of $V P H X[m, n]$

\begin{tabular}{|c|c|c|c|c|}
\hline$\left(\delta_{G}(u), \delta_{G}(v)\right)$ & $(6,8)$ & $(8,8)$ & $(8,9)$ & $(9,9)$ \\
\hline Frequency & $4 m$ & $2 m$ & $2 m$ & $9 m(n-1)$ \\
\hline
\end{tabular}

Theorem 1. The neighborhood Zagreb index $M_{N}$ of $T U C_{4} C_{8}[m, n](m, n \geq 2), T U A C_{6}[m, n], V P H X[m, n]$, and $V P H Y[m, n]$ nanotubes are given by:

(i) $M_{N}\left(T U C_{4} C_{8}[m, n]\right)=648 m n-130 m$,

(ii) $M_{N}\left(T U A C_{6}[m, n]\right)=162 m n-16 m$,

(iii) $M_{N}(V P H X[m, n])=486 m n-158 m$,

(iv) $M_{N}(V P H Y[m, n])=486 m n$.

Proof. The general formula of neighborhood Zagreb index $M_{N}$ is given by:

$$
M_{N}(G)=\sum_{v \in V(G)} \delta_{G}(v)^{2} .
$$

(i) Let $G$ be the $T U C_{4} C_{8}[m, n]$ nanotube for $(m, n \geq 2)$. Then applying the Table 1 on the definition of neighborhood Zagreb index, we obtain:

$$
\begin{aligned}
M_{N}(G) & =\sum_{v \in V_{5}} \delta_{G}(v)^{2}+\sum_{v \in V_{8}} \delta_{G}(v)^{2}+\sum_{v \in V_{9}} \delta_{G}(v)^{2} \\
& =\left|V_{5}\right|\left(5^{2}\right)+\left|V_{8}\right|\left(8^{2}\right)+\left|V_{9}\right|\left(9^{2}\right) \\
& =4 m\left(5^{2}\right)+4 m\left(8^{2}\right)+(8 m n-6 m)\left(9^{2}\right) \\
& =648 m n-130 m .
\end{aligned}
$$


(ii) Let $G$ be the V-phenylenic nanotube $\left(T U A C_{6}[m, n]\right)$. Then applying the Table 3 on the general formula of neighborhood Zagreb index, we have:

$$
\begin{aligned}
M_{N}(G) & =\sum_{v \in V_{5}} \delta_{G}(v)^{2}+\sum_{v \in V_{8}} \delta_{G}(v)^{2}+\sum_{v \in V_{9}} \delta_{G}(v)^{2} \\
& =\left|V_{5}\right|\left(5^{2}\right)+\left|V_{8}\right|\left(8^{2}\right)+\left|V_{9}\right|\left(9^{2}\right) \\
& =2 m\left(6^{2}\right)+2 m\left(8^{2}\right)+2 m(n-1)\left(9^{2}\right) \\
& =486 m n-158 m .
\end{aligned}
$$

(iii) Let $G$ be the V-phenylenic nanotube ( $V P H X[m, n])$. Then applying the Table 5 on the general formula of neighborhood Zagreb index, we have:

$$
\begin{aligned}
M_{N}(G) & =\sum_{v \in V_{6}} \delta_{G}(v)^{2}+\sum_{v \in V_{8}} \delta_{G}(v)^{2}+\sum_{v \in V_{9}} \delta_{G}(v)^{2} \\
& =\left|V_{6}\right|\left(6^{2}\right)+\left|V_{8}\right|\left(8^{2}\right)+\left|V_{9}\right|\left(9^{2}\right) \\
& =2 m\left(6^{2}\right)+4 m\left(8^{2}\right)+(6 m n-6 m)\left(9^{2}\right) \\
& =486 m n-158 m .
\end{aligned}
$$

(iv) Let $G$ be the V-phenylenic nanotori $(V P H Y[m, n])$. Its clear from Fig. 3 that $V(G)=V_{9}$ and $\left|V_{9}\right|=6 m n$. The required result follows clearly from the definition of neighborhood Zagreb index.

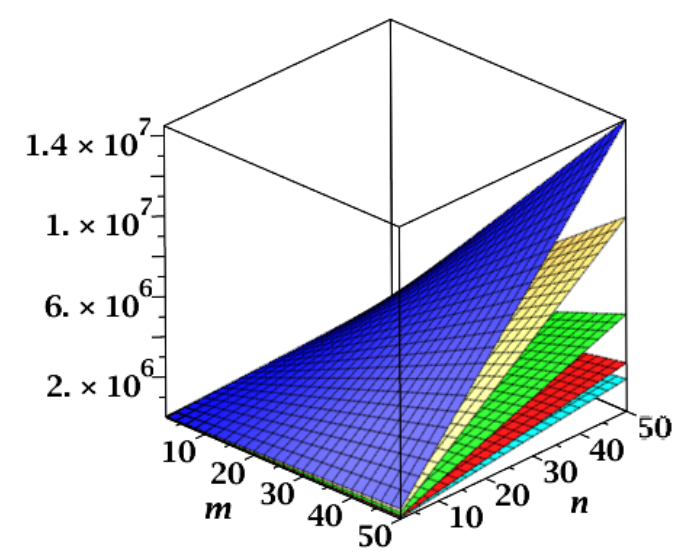

FIG. 5. Topological indices for $T U C_{4} C_{8}[m, n]$ nanotube

Theorem 2. The neighborhood version of Forgotten topological index $F_{N}$ of $T U C_{4} C_{8}[m, n](m, n \geq 2), T U A C_{6}[m, n]$, $V P H X[m, n]$, and $V P H Y[m, n]$ nanotubes are given by:

(i) $F_{N}\left(T U C_{4} C_{8}[m, n]\right)=5832 m n-1826 m$,

(ii) $F_{N}\left(T U A C_{6}[m, n]\right)=1458 m n-184 m$,

(iii) $F_{N}(V P H X[m, n])=4374 m n-1894 m$,

(iv) $F_{N}(V P H Y[m, n])=4374 m n$,

Proof. The general formula of neighborhood version of Forgotten topological index $F_{N}$ is given by:

$$
F_{N}(G)=\sum_{v \in V(G)} \delta_{G}(v)^{3}
$$

(i) Let $G$ be the $T U C_{4} C_{8}[m, n]$ nanotube for $(m, n \geq 2)$. Then applying the Table 1 on the definition of neighborhood version of Forgotten topological index, we obtain:

$$
\begin{aligned}
F_{N}(G) & =\sum_{v \in V_{5}} \delta_{G}(v)^{3}+\sum_{v \in V_{8}} \delta_{G}(v)^{3}+\sum_{v \in V_{9}} \delta_{G}(v)^{3} \\
& =\left|V_{5}\right|\left(5^{3}\right)+\left|V_{8}\right|\left(8^{3}\right)+\left|V_{9}\right|\left(9^{3}\right) \\
& =4 m\left(5^{3}\right)+4 m\left(8^{3}\right)+(8 m n-6 m)\left(9^{3}\right) \\
& =5832 m n-1826 m .
\end{aligned}
$$


(ii) Let $G$ be the V-phenylenic nanotube $\left(T U A C_{6}[m, n]\right)$. Then applying the Table 3 on the general formula of neighborhood version of Forgotten topological index, we have:

$$
\begin{aligned}
F_{N}(G) & =\sum_{v \in V_{5}} \delta_{G}(v)^{3}+\sum_{v \in V_{8}} \delta_{G}(v)^{3}+\sum_{v \in V_{9}} \delta_{G}(v)^{3} \\
& =\left|V_{5}\right|\left(5^{3}\right)+\left|V_{8}\right|\left(8^{3}\right)+\left|V_{9}\right|\left(9^{3}\right) \\
& =2 m\left(5^{3}\right)+2 m\left(8^{3}\right)+2 m(n-1)\left(9^{3}\right) \\
& =1458 m n-184 m .
\end{aligned}
$$

(iii) Let $G$ be the V-phenylenic nanotube (VPHX $[m, n])$. Then applying the Table 5 on the general formula of neighborhood version of Forgotten topological index, we have:

$$
\begin{aligned}
F_{N}(G) & =\sum_{v \in V_{6}} \delta_{G}(v)^{3}+\sum_{v \in V_{8}} \delta_{G}(v)^{3}+\sum_{v \in V_{9}} \delta_{G}(v)^{3} \\
& =\left|V_{6}\right|\left(6^{3}\right)+\left|V_{8}\right|\left(8^{3}\right)+\left|V_{9}\right|\left(9^{3}\right) \\
& =2 m\left(6^{3}\right)+4 m\left(8^{3}\right)+(6 m n-6 m)\left(9^{3}\right) \\
& =4374 m n-1894 m .
\end{aligned}
$$

(iv) Let $G$ be the V-phenylenic nanotori ( $V P H Y[m, n]$ ). From Fig. 3, we have, $V(G)=V_{9}$ and $\left|V_{9}\right|=6 m n$. Using the definition of neighborhood version of Forgotten topological index, the desired result can be obtained easily.

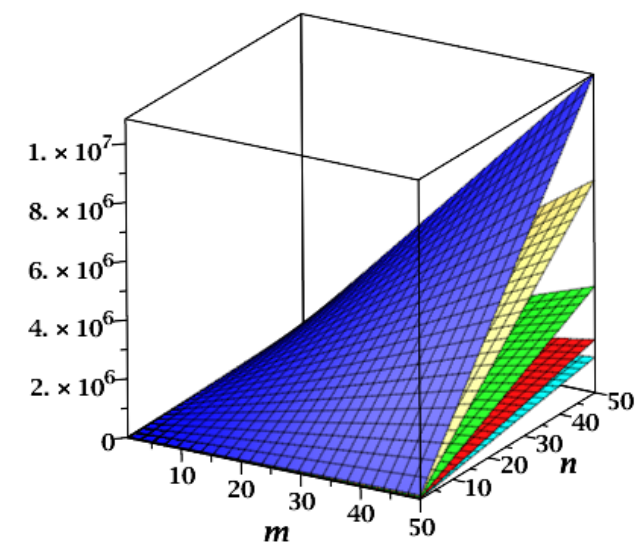

FIG. 6. Topological indices for $V P H X[m, n]$ nanotube.

Theorem 3. The modified neighborhood version of Forgotten topological index $F_{N}^{*}$ of $T U C_{4} C_{8}[m, n](m, n \geq 2)$, $\mathrm{TU}_{A} \mathrm{C}_{6}[\mathrm{~m}, n], \mathrm{VPH} X[\mathrm{~m}, n]$, and $V \mathrm{PHY}[\mathrm{m}, n]$ nanotubes are given by:

(i) $F_{N}^{*}\left(T U C_{4} C_{8}[m, n]\right)=1944 m n-490 m$,

(ii) $F_{N}^{*}\left(T U A C_{6}[m, n]\right)=486 m n-2 m$,

(iii) $F_{N}^{*}(V P H X[m, n])=1458 m n-512 m$,

(iv) $F_{N}^{*}(V P H Y[m, n])=1458 m n$.

Proof. The general formula of modified neighborhood version of Forgotten topological index $F_{N}^{*}$ is given by:

$$
F_{N}^{*}(G)=\sum_{u v \in E(G)}\left[\delta_{G}(u)^{2}+\delta_{G}(v)^{2}\right] .
$$

(i) Let $G$ be the $T U C_{4} C_{8}[m, n]$ nanotube for $(m, n \geq 2)$. Then applying the Table 2 on the definition of modified neighborhood version of Forgotten topological index, we obtain: 


$$
\begin{aligned}
F_{N}^{*}(G)= & \sum_{u v \in E_{(5,5)}}\left[\delta_{G}(u)^{2}+\delta_{G}(v)^{2}\right]+\sum_{u v \in E_{(5,8)}}\left[\delta_{G}(u)^{2}+\delta_{G}(v)^{2}\right]+\sum_{u v \in E_{(8,8)}}\left[\delta_{G}(u)^{2}+\delta_{G}(v)^{2}\right] \\
& +\sum_{u v \in E_{(8,9)}}\left[\delta_{G}(u)^{2}+\delta_{G}(v)^{2}\right]+\sum_{u v \in E_{(9,9)}}\left[\delta_{G}(u)^{2}+\delta_{G}(v)^{2}\right] \\
= & \left|E_{(5,5)}\right|\left(5^{2}+5^{2}\right)+\left|E_{(5,8)}\right|\left(5^{2}+8^{2}\right)+\left|E_{(8,8)}\right|\left(8^{2}+8^{2}\right)+\left|E_{(8,9)}\right|\left(8^{2}+9^{2}\right) \\
& +\left|E_{(9,9)}\right|\left(9^{2}+9^{2}\right) \\
= & 2 m\left(5^{2}+5^{2}\right)+4 m\left(5^{2}+8^{2}\right)+2 m\left(8^{2}+8^{2}\right)+4 m\left(8^{2}+9^{2}\right)+m(12 n-11)\left(9^{2}+9^{2}\right) .
\end{aligned}
$$

After simplification the desired result can be obtained easily.

(ii) Let $G$ be the $T U A C_{6}[m, n]$ nanotube. Then applying the Table 4 on the definition of modified neighborhood version of Forgotten topological index, we obtain:

$$
\begin{aligned}
F_{N}^{*}(G)= & \sum_{u v \in E_{(5,5)}}\left[\delta_{G}(u)^{2}+\delta_{G}(v)^{2}\right]+\sum_{u v \in E_{(5,8)}}\left[\delta_{G}(u)^{2}+\delta_{G}(v)^{2}\right]+\sum_{u v \in E_{(8,8)}}\left[\delta_{G}(u)^{2}+\delta_{G}(v)^{2}\right] \\
& +\sum_{u v \in E_{(8,9)}}\left[\delta_{G}(u)^{2}+\delta_{G}(v)^{2}\right]+\sum_{u v \in E_{(9,9)}}\left[\delta_{G}(u)^{2}+\delta_{G}(v)^{2}\right] \\
= & \left|E_{(5,5)}\right|\left(5^{2}+5^{2}\right)+\left|E_{(5,8)}\right|\left(5^{2}+8^{2}\right)+\left|E_{(8,8)}\right|\left(8^{2}+8^{2}\right)+\left|E_{(8,9)}\right|\left(8^{2}+9^{2}\right) \\
& +\left|E_{(9,9)}\right|\left(9^{2}+9^{2}\right) \\
= & m\left(5^{2}+5^{2}\right)+2 m\left(5^{2}+8^{2}\right)+m\left(8^{2}+8^{2}\right)+2 m\left(8^{2}+9^{2}\right)+m(3 n-4)\left(9^{2}+9^{2}\right) .
\end{aligned}
$$

After simplification the required result can be obtained easily.

(iii) Let $G$ be the $V P H X[m, n]$ nanotube. Then applying the Table 6 on the definition of modified neighborhood version of Forgotten topological index, we obtain:

$$
\begin{aligned}
F_{N}^{*}(G)= & \sum_{u v \in E_{(6,8)}}\left[\delta_{G}(u)^{2}+\delta_{G}(v)^{2}\right]+\sum_{u v \in E_{(8,8)}}\left[\delta_{G}(u)^{2}+\delta_{G}(v)^{2}\right] \\
& +\sum_{u v \in E_{(8,9)}}\left[\delta_{G}(u)^{2}+\delta_{G}(v)^{2}\right]+\sum_{u v \in E_{(9,9)}}\left[\delta_{G}(u)^{2}+\delta_{G}(v)^{2}\right] \\
= & \left|E_{(6,8)}\right|\left(6^{2}+8^{2}\right)+\left|E_{(8,8)}\right|\left(8^{2}+8^{2}\right)+\left|E_{(8,9)}\right|\left(8^{2}+9^{2}\right)+\left|E_{(9,9)}\right|\left(9^{2}+9^{2}\right) \\
= & 4 m\left(6^{2}+8^{2}\right)+2 m\left(8^{2}+8^{2}\right)+2 m\left(8^{2}+9^{2}\right)+9 m(n-1)\left(9^{2}+9^{2}\right) .
\end{aligned}
$$

After simplification the required result can be obtained easily.

(iv) Let $G$ be the $V P H Y[m, n]$ nanotube. From Fig. 3, it is clear that $E(G)=E_{(9,9)}$ and $\left|E_{(9,9)}\right|=9 m n$. Thus, we have $F_{N}^{*}(G)=9 m n\left(9^{2}+9^{2}\right)=1458 m n$

Hence the proof.

Theorem 4. The neighborhood version of second Zagreb index $M_{2}^{*}$ of $T U C_{4} C_{8}[m, n](m, n \geq 2), T U A C_{6}[m, n]$, $V P H X[m, n]$, and $V P H Y[m, n]$ nanotubes are given by:

(i) $M_{2}^{*}\left(T U C_{4} C_{8}[m, n]\right)=972 m n-265 m$,

(ii) $M_{2}^{*}\left(T U A C_{6}[m, n]\right)=243 m n-11 m$,

(iii) $M_{2}^{*}(V P H X[m, n])=729 m n-265 m$,

(iv) $M_{2}^{*}(V P H Y[m, n])=729 m n$.

Proof. The general formula of neighborhood version of second Zagreb index $M_{2}^{*}$ is given by:

$$
M_{2}^{*}(G)=\sum_{u v \in E(G)}\left[\delta_{G}(u) \delta_{G}(v)\right] .
$$

(i) Let $G$ be the $T U C_{4} C_{8}[m, n]$ nanotube for $(m, n \geq 2)$. Then applying the Table 2 on the general form of neighborhood version of second Zagreb index, we obtain the following computation. 


$$
\begin{aligned}
M_{2}^{*}(G)= & \sum_{u v \in E_{(5,5)}}\left[\delta_{G}(u) \cdot \delta_{G}(v)\right]+\sum_{u v \in E_{(5,8)}}\left[\delta_{G}(u) \cdot \delta_{G}(v)\right]+\sum_{u v \in E_{(8,8)}}\left[\delta_{G}(u) \cdot \delta_{G}(v)\right] \\
& +\sum_{u v \in E_{(8,9)}}\left[\delta_{G}(u) \cdot \delta_{G}(v)\right]+\sum_{u v \in E_{(9,9)}}\left[\delta_{G}(u) \cdot \delta_{G}(v)\right] \\
= & \left|E_{(5,5)}\right|(5.5)+\left|E_{(5,8)}\right|(5.8)+\left|E_{(8,8)}\right|(8.8)+\left|E_{(8,9)}\right|(8.9)+\left|E_{(9,9)}\right|(9.9) \\
= & 2 m(5.5)+4 m(5.8)+2 m(8.8)+4 m(8.9)+(12 m n-11 m)(9.9) .
\end{aligned}
$$

After simplification, the desired result can be easily obtained.

(ii) Let $G$ be the $T U A C_{6}[m, n]$ nanotube. Then, applying the Table 4 on the definition of neighborhood version of second Zagreb index, we get the following derivation:

$$
\begin{aligned}
M_{2}^{*}(G)= & \sum_{u v \in E_{(5,5)}}\left[\delta_{G}(u) \cdot \delta_{G}(v)\right]+\sum_{u v \in E_{(5,8)}}\left[\delta_{G}(u) \cdot \delta_{G}(v)\right]+\sum_{u v \in E_{(8,8)}}\left[\delta_{G}(u) \cdot \delta_{G}(v)\right] \\
& +\sum_{u v \in E_{(8,9)}}\left[\delta_{G}(u) \cdot \delta_{G}(v)\right]+\sum_{u v \in E_{(9,9)}}\left[\delta_{G}(u) \cdot \delta_{G}(v)\right] \\
= & \left|E_{(5,5)}\right|(5.5)+\left|E_{(5,8)}\right|(5.8)+\left|E_{(8,8)}\right|(8.8)+\left|E_{(8,9)}\right|(8.9)+\left|E_{(9,9)}\right|(9.9) \\
= & 4 m(6.8)+2 m(8.8)+2 m(8.9)+9 m(n-1)(9.9) \\
= & 243 m n-11 m .
\end{aligned}
$$

(iii) Let $G$ be the $V P H X[m, n]$ nanotube. Then, applying the Table 6 on the definition of neighborhood version of second Zagreb index, we get the following derivation:

$$
\begin{aligned}
M_{2}^{*}(G)= & \sum_{u v \in E_{(6,8)}}\left[\delta_{G}(u) \cdot \delta_{G}(v)\right]+\sum_{u v \in E_{(8,8)}}\left[\delta_{G}(u) \cdot \delta_{G}(v)\right] \\
& +\sum_{u v \in E_{(8,9)}}\left[\delta_{G}(u) \cdot \delta_{G}(v)\right]+\sum_{u v \in E_{(9,9)}}\left[\delta_{G}(u) \cdot \delta_{G}(v)\right] \\
= & \left|E_{(6,8)}\right|(6.8)+\left|E_{(8,8)}\right|(8.8)+\left|E_{(8,9)}\right|(8.9)+\left|E_{(9,9)}\right|(9.9) \\
= & 4 m(6.8)+2 m(8.8)+2 m(8.9)+9 m(n-1)(9.9) \\
= & 729 m n-265 m .
\end{aligned}
$$

(iv) Let $G$ be the $\operatorname{VPHY}[m, n]$ nanotube. From Fig. 3, it is clear that $E(G)=E_{(9,9)}$ and $\left|E_{(9,9)}\right|=9 m n$. Thus, we have $F_{N}^{*}(G)=9 m n(9.9)=729 m n$.

Hence the proof.

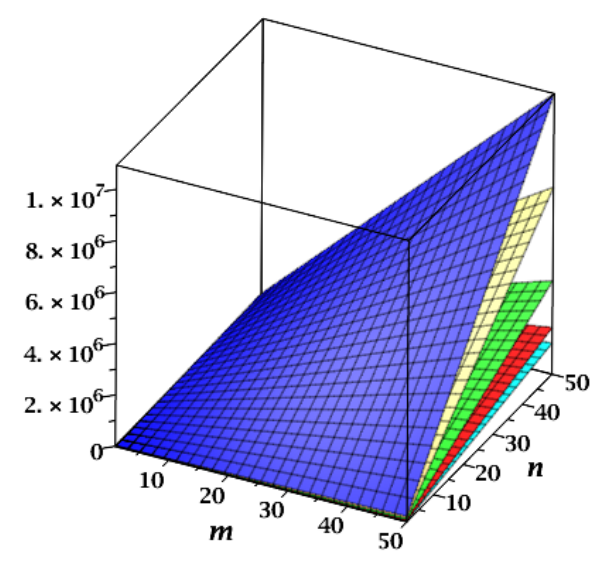

FIG. 7. Topological indices for $V P H Y[m, n]$ nanotori. 
Theorem 5. The neighborhood version of hyper Zagreb index $H M_{N}$ of $T U C_{4} C_{8}[m, n](m, n \geq 2), T U A C_{6}[m, n]$, $V P H X[m, n]$, and $V P H Y[m, n]$ nanotubes are given by:

(i) $H M_{N}\left(T U C_{4} C_{8}[m, n]\right)=3888 m n-1020 m$,

(ii) $H M_{N}\left(T U A C_{6}[m, n]\right)=972 m n-24 m$,

(iii) $H M_{N}(V P H X[m, n])=2916 m n-1042 m$,

(iv) $H M_{N}(V P H Y[m, n])=2916 m n$.

Proof. The general formula of neighborhood version of hyper Zagreb index $H M_{N}$ is given by:

$$
H M_{N}(G)=\sum_{u v \in E(G)}\left[\delta_{G}(u)+\delta_{G}(v)\right]^{2} .
$$

(i) Let $G$ be the $T U C_{4} C_{8}[m, n]$ nanotube for $(m, n \geq 2)$. Then applying Table 2 on the general form of neighborhood version of hyper Zagreb index, we obtain the following computation:

$$
\begin{aligned}
H M_{N}(G)= & \sum_{u v \in E_{(5,5)}}\left[\delta_{G}(u)+\delta_{G}(v)\right]^{2}+\sum_{u v \in E_{(5,8)}}\left[\delta_{G}(u)+\delta_{G}(v)\right]^{2}+\sum_{u v \in E_{(8,8)}}\left[\delta_{G}(u)+\right. \\
& \left.\delta_{G}(v)\right]^{2}+\sum_{u v \in E_{(8,9)}}\left[\delta_{G}(u)+\delta_{G}(v)\right]^{2}+\sum_{u v \in E_{(9,9)}}\left[\delta_{G}(u)+\delta_{G}(v)\right]^{2} \\
= & \left|E_{(5,5)}\right|(5+5)^{2}+\left|E_{(5,8)}\right|(5+8)^{2}+\left|E_{(8,8)}\right|(8+8)^{2}+\left|E_{(8,9)}\right|(8+9)^{2} \\
& +\left|E_{(9,9)}\right|(9+9)^{2} \\
= & 2 m(5+5)^{2}+4 m(5+8)^{2}+2 m(8+8)^{2}+4 m(8+9)^{2}+(12 m n-11 m)(9+9)^{2} .
\end{aligned}
$$

After simplification the desired result can be obtained easily.

(ii) Let $G$ be the $T U A C_{6}[m, n]$ nanotube. Then applying the Table 4 on the definition of neighborhood version of second Zagreb index, we get the following derivation:

$$
\begin{aligned}
H M_{N}(G)= & \sum_{u v \in E_{(5,5)}}\left[\delta_{G}(u)+\delta_{G}(v)\right]^{2}+\sum_{u v \in E_{(5,8)}}\left[\delta_{G}(u)+\delta_{G}(v)\right]^{2}+\sum_{u v \in E_{(8,8)}}\left[\delta_{G}(u)+\delta_{G}(v)\right]^{2} \\
& +\sum_{u v \in E_{(8,9)}}\left[\delta_{G}(u)+\delta_{G}(v)\right]^{2}+\sum_{u v \in E_{(9,9)}}\left[\delta_{G}(u)+\delta_{G}(v)\right]^{2} \\
= & \left|E_{(5,5)}\right|(5+5)^{2}+\left|E_{(5,8)}\right|(5+8)^{2}+\left|E_{(8,8)}\right|(8+8)^{2}+\left|E_{(8,9)}\right|(8+9)^{2}+\left|E_{(9,9)}\right| \\
& (9+9)^{2} \\
= & 4 m(5+5)^{2}+4 m(5+8)^{2}+2 m(8.8)+2 m(8+9)^{2}+9 m(n-1)(9+9)^{2} \\
= & 972 m n-24 m .
\end{aligned}
$$

(iii) Let $G$ be the $V P H X[m, n]$ nanotube. Then applying the Table 6 on the definition of neighborhood version of second Zagreb index, we get the following derivation:

$$
\begin{aligned}
H M_{N}(G)= & \sum_{u v \in E_{(6,8)}}\left[\delta_{G}(u)+\delta_{G}(v)\right]^{2}+\sum_{u v \in E_{(8,8)}}\left[\delta_{G}(u)+\delta_{G}(v)\right]^{2}+\sum_{u v \in E_{(8,9)}}\left[\delta_{G}(u)\right. \\
& \left.+\delta_{G}(v)\right]^{2}+\sum_{u v \in E_{(9,9)}}\left[\delta_{G}(u)+\delta_{G}(v)\right]^{2} \\
= & \left|E_{(6,8)}\right|(6+8)^{2}+\left|E_{(8,8)}\right|(8+8)^{2}+\left|E_{(8,9)}\right|(8+9)^{2}+\left|E_{(9,9)}\right|(9+9)^{2} \\
= & 4 m(6+8)^{2}+2 m(8.8)+2 m(8+9)^{2}+9 m(n-1)(9+9)^{2} \\
= & 2916 m n-1042 m .
\end{aligned}
$$

(iv) Let $G$ be the $\operatorname{VPHY}[m, n]$ nanotube. From Fig. 3, it is clear that $E(G)=E_{(9,9)}$ and $\left|E_{(9,9)}\right|=9 m n$. Thus, we have $F_{N}^{*}(G)=9 m n(9+9)^{2}=2916 m n$

Hence the proof. 


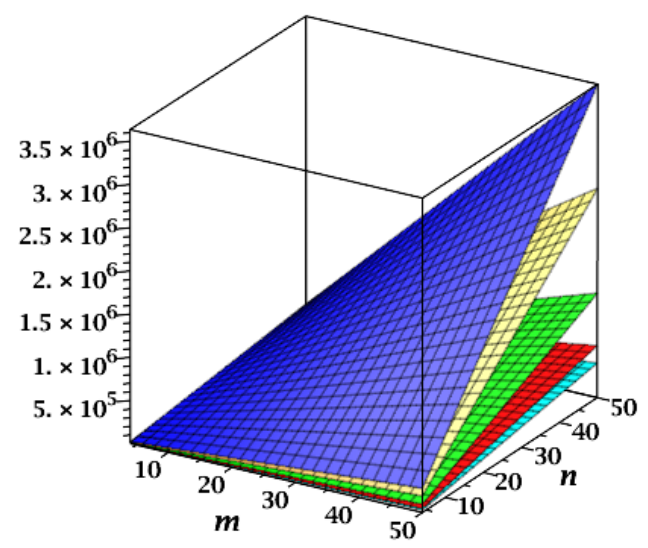

FIG. 8. Topological indices for $T U A C_{6}[m, n]$ nanotube.

The surface plotting of topological indices for the nanotubes and nanotori are shown in the Figs. 5, 6, 7, and 8. We have built the figures using Maple 2015.1 software taking the parametric values $(m, n)$ in $[2,50]$. For different indices, different colors are used. We put cyan, blue, green, red and gold colors for $M_{N}, F_{N}, F_{N}^{*}, M_{2}^{*}$, and $H M_{N}$ indices respectively.

\section{Remarks and conclusion}

In this article, the structures of $T U R C_{4} C_{8}(S)$, armchair polyhex nanotube $T U A C_{6}, \mathrm{~V}$-phenylenic nanotube $V P H X[m, n]$, and V-phenylenic nanotori $V P H Y[m, n]$ are discussed and explicit expressions of $M_{N}, F_{N}, F_{N}^{*}$, $M_{2}^{*}$, and $H M_{N}$ are derived for them. In fact, comparison among these indices for the considered nanotubes and nanotori are shown in the Figs. 5,6,7, and 8. Clearly, the indices for different nanotubes and nanotori are growing in the following order.

$$
T U A C_{6}[m, n]<V P H Y[m, n]<V P H X[m, n]<T U R C_{4} C_{8}(S),
$$

where in each case, indices have following order.

$$
M_{N}<M_{2}^{*}<F_{N}^{*}<H M_{N}<F_{N} .
$$

Thus, for each structure discussed above, the indices behave somewhat differently. The formulas obtained here enable the chemical structure of nano structures to be correlated with a large amount of information about their physicochemical characteristics.

\section{Acknowledgments}

The first author is very obliged to the Department of Science and Technology (DST), Government of India for the Inspire Fellowship [IF170148].

\section{References}

[1] Trinajstić N. Chemical Graph Theory. CRC Press, Boca Raton, 1983.

[2] Gutman I. Polansky O.E. Mathematical Concepts in Organic Chemistry. Springer, Berlin, 1986.

[3] Diudea M.V., Gutman I., Lorentz J. Molecular Topology. Romania: Babes-Bolyai University, 2001.

[4] Gutman I. Degreebased topological indices. Croat. Chem. Acta, 2013, 86 (4), P. 351-361.

[5] Gutman I., Trinajstić N. Graph theory and molecular orbitals.Total $\pi$-electron energy of alternate hydrocarbons. Chem. Phys. Lett., 1972, 17, P. $535-538$.

[6] Gutman I., Das K.C. The first Zagreb index 30 years after. MATCH Commun. Math. Comput. Chem., 2004, 50, P. 83-92.

[7] Furtula B., Gutman I. A forgotten topological index. J. Math. Chem., 2015, 53 (4), P. 1184-1190.

[8] Shirdel G.H., Rezapour H., Sayadi A.M. The hyper Zagreb index of graph operations. Iran. J. Math. Chem., 2013,4 (2), P. $213-220$.

[9] Mondal S., De N., Pal A. On neighbourhood zagreb index of product graphs. arXiv:1805.05273, 2018.

[10] Mondal S., De N., Pal A. On some new neighbourhood degree based indices. Acta Chemica Iasi, 2019,27 (1), P. $31-46$.

[11] Gao Y.Y., Sardar M.S., Hosamani S.M., Farahani M.R. Computing sanskruti index of TURC4C8(s) nanotube. Int. J. Pharm. Sci. Res., 2017, 8 (10), P. 4423-4425.

[12] Jiang H., Sardar M.S., et al. Computing sanskruti index V-phenylenic nanotube and nanotori. Int. J. Pure Appl. Math., 2017, 115 (4), P. 859865.

[13] Farahani M.R. Computing $G A_{5}$ index of armchair polyhex nanotube. Le Matematiche, 2014, LXIX, P. 69-76. 
[14] Mondal S., De N., Pal A. On Some New Neighborhood Degree-Based Indices for Some Oxide and Silicate Networks. J. Multidisciplinary Scientific Journal, 2019, 2 (3), P. 384-409.

[15] Kawun Y.C., Munir M., et al. Computational Analysis of topological indices of two Boron Nanotubes. Scientific Reports, 2018,8 (1), 14843.

[16] Kawun Y.C., Munir M., et al. M-Polynomials and topological indices of V-Phenylenic Nanotubes and Nanotori. Scientific Reports, 2017, 7 (1), 8756.

[17] Farahani M. R. On the fourth atom bond connectivity index of armchair polyhex nanotube. Proc. Rom. Acad., Series B, 2013 , 15 (1), P. 3-6.

[18] Ashrafi A.R., Doslic T., Saheli M. The eccentric connectivity index of $T U C_{4} C_{8}(R)$ nanotubes. MATCH Commun. Math. Comput. Chem., 2011, 65, P. 221-230.

[19] Farahani M.R. Fifth Geometric-Arithmetic Index of TURC4C8(S) Nanotubes. J. Chem. Acta, 2013,2 (1), P. $62-64$.

[20] De N., Nayeem S.M.A. Computing the F-index of nanostar dendrimers. Pacific Science Review A: Natural Science and Engineering, 2016, 10.1016/j.psra.2016.06.001.

[21] Gao W., Siddiqui M.K. Molecular Descriptors of Nanotube, Oxide, Silicate, and Triangulene Networks. J. Chem., $2017,6540754$. 